\title{
Extra-virgin olive oil polyphenols inhibit HER2 (erbB-2)-induced malignant transformation in human breast epithelial cells: Relationship between the chemical structures of extra-virgin olive oil secoiridoids and lignans and their inhibitory activities on the tyrosine kinase activity of HER2
}

\author{
JAVIER A. MENENDEZ ${ }^{1,2,3}$, ALEJANDRO VAZQUEZ-MARTIN ${ }^{1,2,3}$, CRISTINA OLIVERAS-FERRAROS ${ }^{1,2,3}$ \\ ROCIO GARCIA-VILLALBA ${ }^{1,4}$, ALEGRIA CARRASCO-PANCORBO ${ }^{4}$, \\ ALBERTO FERNANDEZ-GUTIERREZ ${ }^{4}$ and ANTONIO SEGURA-CARRETERO ${ }^{4}$ \\ ${ }^{1}$ Catalan Institute of Oncology (ICO)-Health Services Division of Catalonia; ${ }^{2}$ Girona Biomedical \\ Research Institute (IdIBGi); ${ }^{3}$ Medical Oncology, Dr Josep Trueta University Hospital of Girona, Girona, \\ Catalonia; ${ }^{4}$ Department of Analytical Chemistry, Faculty of Sciences, University of Granada, Granada, Spain
}

Received August 11,2008; Accepted October 7, 2008

DOI: 10.3892/ijo_00000127

\begin{abstract}
Depending on their structure, some polyphenols (e.g. flavonoids) abundantly found in plant-derived beverages such as green tea can efficiently inhibit tyrosine kinase and serine/threonine kinase activities. Extra-virgin olive oil (EVOO - the juice of the olive obtained solely by pressing and consumed without any further refining process) is unique among other vegetable oils because of the high level of naturally occurring phenolic compounds. We explored the ability of EVOO polyphenols to modulate HER2 tyrosine kinase receptor-induced in vitro transformed phenotype in human breast epithelial cells. Using MCF10A normal breast epithelial cells retrovirally engineered to overexpress the wild-type sequence of human HER2, we further determined the relationship between chemical structures of EVOO-derived phenolics and their inhibitory activities on the tyrosine kinase activity of the HER2 oncoprotein. When the activation (phosphorylation) status of HER2 was semi-quantitatively measured the secoiridoids blocked HER2 signaling by rapidly reducing the activation status of the 1248 tyrosine residue
\end{abstract}

Correspondence to: Dr Javier A. Menendez, Catalan Institute of Oncology (ICO), Girona Biomedical Research Institute (IdIBGi), Medical Oncology, Dr Josep Trueta University Hospital of Girona, Avenida de Francia s/n, E-17007 Girona, Catalonia, Spain E-mail: jmenendez@ico.scs.es

Dr Antonio Segura-Carretero, Research Group FQM-297, Department of Analytical Chemistry, Faculty of Sciences, University of Granada, C/Fuentenueva s/n, E-18071 Granada, Spain E-mail: ansegura@ugr.es

Key words: olive oil, Mediterranean diet, phenolics, breast cancer, HER2, tyrosine kinases
(Y1248), the main autophosphorylation site of HER2. EVOO-derived single phenols tyrosol and hydroxytyrosol and the phenolic acid elenolic acid failed to significantly decrease HER2 tyrosine kinase activity. The anti-HER2 tyrosine kinase activity $\mathrm{IC}_{50}$ values were up to 5-times lower in the presence of EVOO-derived lignans and secoiridoids than in the presence of EVOO-derived single phenols and phenolic acids. EVOO polyphenols induced strong tumoricidal effects by selectively triggering high levels of apoptotic cell death in HER2-positive MCF10A/HER2 cells but not in MCF10A/pBABE matched control cells. EVOO lignans and secoiridoids prevented HER2-induced in vitro transformed phenotype as they inhibited colony formation of MCF10A/HER2 cells in soft-agar. Our current findings not only molecularly support recent epidemiological evidence revealing that EVOO-related anti-breast cancer effects primarily affect the occurrence of breast tumors overexpressing the type I receptor tyrosine kinase HER2 but further suggest that the stereochemistry of EVOO-derived lignans and secoiridoids might provide an excellent and safe platform for the design of new HER2 targeted anti-breast cancer drugs.

\section{Introduction}

The final proof about the specific mechanisms by which the different components of olive oil (OO) exert their potential protective effects on the promotion and progression of human breast cancer is a major challenge that is beginning to be addressed $(1,2)$. However, it appears that specific components of OO may influence the genetic program that drives breast cancer development and progression (3-6). In this regard, our experimental approach in the last few years has suggested that OO-related anti-breast cancer actions mainly affect the occurrence, the aggressive behavior and the therapeutic management of HER2 oncogene-driven breast carcinomas: 
First, a high-OO diet influenced negatively DMBA-induced formation of tumors in the mammary gland of female Sprague-Dawley rats by modifying the mRNA expression levels of neu (HER2) $(7,8)$. Second, exogenous supplementation with physiological concentrations of oleic acid (OA; 18:1n-9), the main monounsaturated fatty acid in OO, was found to drastically suppress the expression of HER2 at the transcriptional level when using human breast cancer-derived in vitro models naturally exhibiting HER2 gene amplification and HER2 protein overexpression (9-12). Third, the polyphenol oleuropein aglycone - a bitter principle abundantly found in extra-virgin olive oil (EVOO; the juice of the olive obtained solely by pressing and consumed without any further refining process), significantly reversed breast cancer acquired autoresistance to the anti-HER2 monoclonal antibody trastuzumab (Herceptin ${ }^{\mathrm{TM}}$ ) (13). Importantly, it has been recently suggested that the effects of the OO-rich Mediterranean diet on breast cancer risk might be underestimated when HER2 oncogene-positive and HER2-negative breast carcinomas are considered together (14). Therefore, basic research and epidemiological studies begin to support the notion that EVOO-related anti-breast cancer actions primarily affect the occurrence, the aggressive behavior and the therapeutic management of breast tumors overexpressing the type I receptor tyrosine kinase (RTK) HER2, an oncogene that is amplified and/or overexpressed in 20 to $30 \%$ of breast carcinomas and is associated with unfavorable prognosis, shorter relapse time and decreased overall survival (15-19).

The chemical composition of $\mathrm{OO}$ is principally OA-rich glycerols representing $>98 \%$ of the OO total weight $(20,21)$. Minor components amount to $\sim 2 \%$ of the total OO weight and include, among others, aliphatic and triterpenic alcohols, sterols, hydrocarbons, volatile compounds and several antioxidants. Although tocopherols and carotenes are also present, hydrophilic phenolics represent the most abundant family of OO antioxidants. Specifically, EVOO is unique among other vegetable oils because of the high level of phenolic compounds (22-30). These levels are possible because it is obtained from the olive fruit (Olea Europea L.) solely by mechanical means, without further treatment other than washing, filtration, decantation or centrifugation. This process retains minor compounds originally present in the olive fruit, which are usually removed from other vegetable oils during various stages of refining. Among the various components of the unsaponifiable fraction of the EVOO, phenol compounds are the most important for their contribution to the sensory characteristics and the shelf life of OO. The main components of the phenolic fraction of EVOO are hydroxytyrosol, tyrosol, and their derivatives linked to the aldehydic and dialdehydic forms of elenolic acid, which are described as secoiridoids (that include aglycone derivatives of oleuropein, dimethyloleuropein and ligstroside, which are also present in olive fruit). Moreover, significant amounts of lignans, such as (+)-pinoresinol and 1-(+)-acetoxypinoresinol, are also present, as well as flavonoids (luteolin and apigenin), phenolic acids (such as caffeic, vanillic, syringic, $p$-coumaric, $o$-coumaric, protocatechuic, sinapic and $p$-hydroxybenzoic acid) and hydroxy-isochromans. The exact composition of phenolics compounds in EVOO is related to agronomic and technological aspects.
Depending on their structure, some naturally occurring polyphenols (e.g. flavonoids) found in abundance in plantderived beverages such as green tea can efficiently inhibit tyrosine kinase and serine/threonine kinase activities, which may account for the epidemiologically observed inverse correlation between green-tea drinking and cancer risk in Oriental populations (31-36). Here, we hypothesized that EVOO-derived phenolics might decrease the activation status of the HER2 tyrosine kinase receptor and, hence, to inhibit HER2-induced in vitro transformed phenotype in human breast epithelial cells.

\section{Materials and methods}

Materials. A 50/50 mixture of two commercial EVOO samples (i.e. Picual and Arbequina Spanish varieties) was used for this study. Solid-phase extraction (SPE) followed by semipreparative high-performance liquid chromatography (HPLC) was used to isolate phenolic fractions from commercial EVOO as previously described (37-42). Analytical capillary electrophoresis coupled to mass spectrometry (CE-MS) was performed to check for the composition of the isolated fractions and to confirm their molecular identity. For experimental use EVOO phenolics were prepared freshly from stock solutions (10 mM in $100 \%$ ethanol) and diluted with growth medium. Control cells were cultured in medium containing the same concentration $(\mathrm{v} / \mathrm{v})$ as the experimental cultures with treatments. The vehicle solutions had no noticeable influence on the proliferation of experimental cells.

Cell lines and cell culture conditions. The MCF10A human mammary epithelial cell line (American Type Culture Collection, Manassas, VA, USA) was maintained in DMEM/ F12 medium (Gibco ${ }^{\circledR}$ Cell Culture Systems, Invitrogen Corporation, Carlsbad, CA, USA) containing 5\% horse serum (Invitrogen Corporation), $0.5 \mu \mathrm{g} / \mathrm{ml}$ hydrocortisone (Sigma-Chemicals, St. Louis, MO), $100 \mathrm{ng} / \mathrm{ml}$ cholera toxin (Sigma-Chemicals), $10 \mu \mathrm{g} / \mathrm{ml}$ insulin (Sigma-Chemicals), $2.5 \mathrm{mM}$ L-glutamine (Biofluids, Biosource International, Camarillo, CA) and $20 \mathrm{ng} / \mathrm{ml}$ EGF (Sigma-Chemicals). Cells were maintained at $37^{\circ} \mathrm{C}$ in a humidified atmosphere of $95 \%$ air and $5 \% \mathrm{CO}_{2}$. Cells were screened periodically for Mycoplasma contamination.

Construction of $p B A B E / H E R 2$ retroviruses and retroviral infection of MCF10A cells. A full-length human HER2 cDNA construct in the pCMV-SPORT6 plasmid was purchased from RZPD (Berlin, Germany). The insert was excised from pCMV-SPORT6 using EcoRV and NotI sites and blunt end ligated into the pBABE-puro retroviral vector (Addgene) at the EcoRI site. Retroviruses were generated by co-transfection of 293T-derived phoenix cells with the retroviral constructs (pBABE, pBABE-HER2) and the packaging vector pCL-Eco by using FuGene transfection reagent (Roche Diagnostics) g of each plasmid per $0.5 \times 10^{6}$ cells. 293T cells were Barcelona, Spain) and 5 cultured at $5 \% \mathrm{CO}_{2}, 37^{\circ} \mathrm{C}$ in DMEM containing $10 \%(\mathrm{v} / \mathrm{v})$ heat-inactivated FBS. After $48 \mathrm{~h}$, medium conditioned by transfected $293 \mathrm{~T}$ cells was filtered and immediately added to MCF10A cells in the presence of $4 \mu \mathrm{g} / \mathrm{ml}$ polybrene (Sigma-Chemicals). At $48 \mathrm{~h}$ following infection, 
MCF10A/pBABE and MCF10A/HER2 cells were selected by using $2.5 \mu \mathrm{g} / \mathrm{ml}$ puromycin for $72 \mathrm{~h}$. Expression of virally-encoded HER2 protein was confirmed by HER2specific ELISA analyses (see below).

HER2-specific enzyme-linked immunosorbent assay. Determination of HER2 protein content was performed with a commercially available quantitative ELISA (Oncogene Science, Bayer Diagnostics) according to the manufacturer's protocol. To assess the effects of EVOO phenolics on HER2 protein concentrations, breast cancer cells, after a $24 \mathrm{~h}$ starvation period in media without serum, were incubated with graded concentrations of EVOO phenolics. After treatment (48 h), cells were washed twice with cold-PBS and then lysed in buffer $(20 \mathrm{mM}$ Tris- $\mathrm{HCl}, 150 \mathrm{mM} \mathrm{NaCl}, 1 \mathrm{mM}$ EDTA, 1 mM EGTA, 1\% Triton X-100, 2.5 mM sodium pyrophosphate, $1 \mathrm{mM}$ B-glycerophosphate, $1 \mathrm{mM} \mathrm{Na}_{3} \mathrm{VO}_{4}$, $1 \mu \mathrm{g} / \mathrm{ml}$ leupeptin and $1 \mathrm{mM}$ phenylmethylsulfonylfluoride) for $30 \mathrm{~min}$ on ice. The lysates were cleared by centrifugation in an Eppendorff tube $\left(15 \mathrm{~min}\right.$ at $\left.14,000 \mathrm{x} \mathrm{g}, 4^{\circ} \mathrm{C}\right)$. Protein content was determined against a standardized control using the Pierce Protein Assay kit (Rockford, IL, USA). 1:50, 1:500; 1: 5,000 and 1:10,000 dilutions of total cell lysates from EVOO phenolics-treated and control untreated cells were used to quantitate HER2 protein expression in cell cultures. A standard curve was generated by using standard solutions as per manufacturer's instructions. The concentrations of HER2 in test samples (in nanograms of HER2 per milligram of total protein) were determined by interpolation of the sample absorbances from the standard curve. Each experiment was performed in duplicate wells.

Activation status of HER2. The activation (phosphorylation) status of HER2 was measured using the Fast Activated Cellbased ELISA (FACE) kit (Active Motif; Carlsbad, CA, USA) as per manufacturer's instructions. The FACE ErbB-2 (Y1248) kit contains a phosphor-HER2 antibody that was raised in rabbit against a synthetic phosphor-peptide corresponding to residues surrounding phosphorylated Tyr1248 of human HER2 and recognizes HER2 only when phosphorylated at Tyr1248.

Apoptosis assays. The ability of EVOO-derived phenolics to induce apoptosis was assessed using the Cell Death Detection ELISA $^{\text {PLUS }}$ kit obtained from Roche Diagnostics (Barcelona, Spain). Briefly, cells (5 to $10 \times 10^{3} /$ well) were grown in 96-well plates and treated, in duplicates, for $72 \mathrm{~h}$ with the indicated doses of EVOO phenols, as specified. After treatment, the 96-well plates were centrifuged $(200 \mathrm{x} \mathrm{g})$ for $10 \mathrm{~min}$. The supernatant was discharged, lysis buffer was added and samples were incubated at room temperature (RT) for $30 \mathrm{~min}$ following the manufacturer's instructions. Anti-histone biotin and anti-DNA peroxidase antibodies were added to each well and incubated at RT for $2 \mathrm{~h}$. After three washes, the peroxidase substrate was added to each well and the plates were read at $405 \mathrm{~nm}$ at multiple time intervals. The enrichment of histoneDNA fragments in treated cells was expressed as fold increase in absorbance as compared with control (vehicle-treated) cells.
Soft agar colony formation assays. A bottom layer of $1 \mathrm{ml} 2 \mathrm{X}$ complete DMEM/F12 medium containing 0.7\% agar and 10\% horse serum was prepared was prepared in $35 \mathrm{~mm}$ multi-well cluster dishes. After the bottom layers solidified (10,000 cells/ dish) were added in a $1 \mathrm{ml}$ top layer of $2 \mathrm{X}$ complete DMEM/ F12 medium containing $0.35 \%$ agar and $10 \%$ horse serum. All samples were prepared in triplicate. Dishes were incubated in a humidified $5 \% \mathrm{CO}_{2}$ incubator at $37^{\circ} \mathrm{C}$ and colonies measuring $\geq 50 \mu \mathrm{m}$ were counted after $\sim 14$ days after staining with nitroblue tetrazolium (Sigma-Chemicals).

Statistics. Two-group comparisons were performed by the Student's t-test for paired and unpaired values. Comparisons of means of 3 groups were performed by ANOVA and the existence of individual differences, in case of significant $\mathrm{F}$ values at ANOVA, tested by Scheffé's multiple contrasts.

\section{Results}

Generation of HER2-overexpressing MCF10A breast epithelial cells. In the present study, MCF10A cells were engineered to overexpress the wild-type form of human HER2 by retroviral infection, thus avoiding undesirable clone-related effects. When HER2-specific ELISA was employed to quantitatively measure the concentration of HER2 following retroviral infection and stable selection, MCF10A/HER2 cells were found to express HER2 protein levels $~ 35$ times higher than MCF10A/pBABE matched control cells. Thus, cell lysates from empty vector-transduced MCF10A/pBABE cells and MCF10A/HER2 cells were found to stably express $60 \mathrm{ng}$ HER2 $\mathrm{mg}_{\text {protein }}{ }^{-1}$ and 2,200 $\mathrm{ng}$ HER2 $\mathrm{mg}$ protein $^{-1}$, respectively (Fig. 1).

EVOO-derived phenolics inhibit HER2 tyrosine kinase activity. We first evaluated whether short-term exposure to EVOO phenolics (i.e. $6 \mathrm{~h}$ ) altered HER2 tyrosine kinase activity in MCF10A/HER2 cells. The active/inactive status of the main autophosphorylation site of HER2 [i.e. the 1248 tyrosine residue (Tyr1248)] was semi-quantitatively assessed by using the FACE ErbB-2 (Y1248) kit. Forced expression of HER2 tyrosine kinase receptor increased by $\sim 5.0$-fold the baseline HER2-associated tyrosine kinase activity occurring in MCF10A control cells (Fig. 1). Treatment of MCF10A/HER2 cells with EVOO-derived single phenols and phenolic acids significantly decreased HER2 tyrosine kinase activity up to $50 \%$ in the presence of hydroxytyrosol (Fig. 2a). Remarkably, the hyperactive status of HER2 in MCF10A/HER2 cells markedly decreased by $>70 \%$ following short-term treatments with graded concentrations of the EVOO lignan 1-(+)acetoxypinoresinol (Fig. 2b). All the EVOO secoiridoids were capable of significantly reducing HER2 tyrosine kinase activity by $>50 \%$, with a maximal reduction of $\sim 70 \%$ occurring in the presence of ligstroside aglycone (Fig. 2c).

Microscopic analyses revealed that loss of HER2 tyrosine kinase activity upon exogenous supplementation with EVOO phenolics significantly impacted cell morphology of HER2overexpressing MCF10A/HER2 cells, which became already visible at $24 \mathrm{~h}$. When compared with untreated cells, MCF10A/HER2 cells treated with (+)-pinoresinol, DAOA, ligstroside aglycone and oleuropein aglycone underwent 

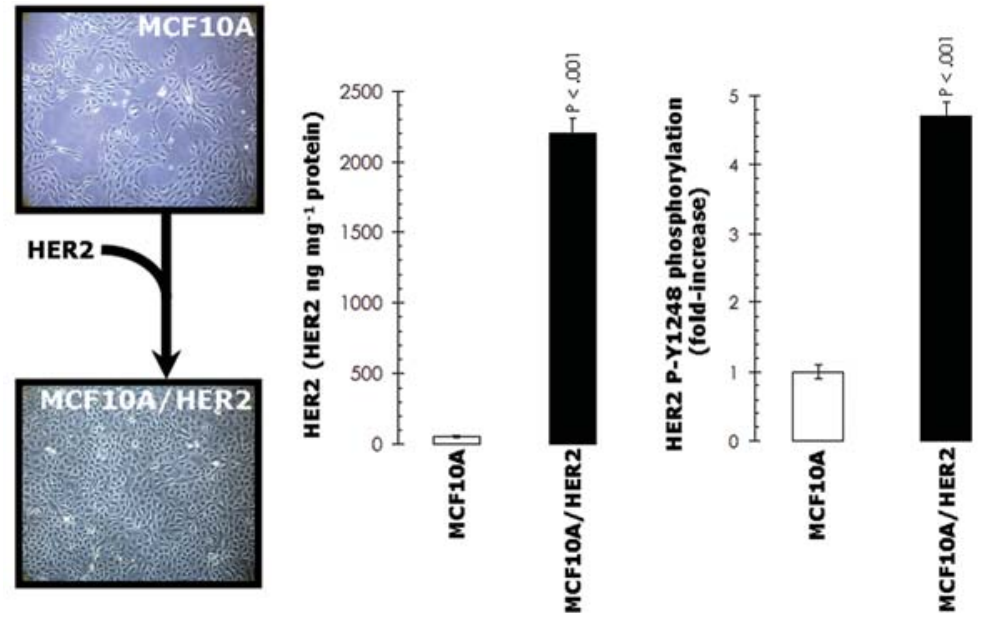

Figure 1. Ectopic overexpression of HER2 in MCF10A cells. MCF10A cells were engineered to overexpress human HER2 as described in 'Materials and methods'. Determination of HER2 protein content in MCF10A/HER2 and MCF10A/pBABE matched control cells was performed with a commercially available quantitative ELISA (Oncogene Science, Bayer Diagnostics) according to the manufacturer's protocol. Assessment of the active/inactive status of the HER2 tyrosine kinase receptor was performed by semi-quantitatively determining the degree of degree of phosphorylation of the 1248 tyrosine residue (Tyr1248) of HER2 by using the FACE ErbB-2 (Y1248) kit as described in 'Materials and methods'. The measurement of phosphor-HER2 (Y1248) in untreated HER2-negative MCF10A cells was arbitrarily designed as 1.0-fold. Results are means (columns) and 95\% confidence intervals (bars) of three independent experiments made in triplicate. Statistically significant differences (one-factor ANOVA analysis) between experimental conditions and unsupplemented control cells are labeled. All statistical tests were two-sided.

a
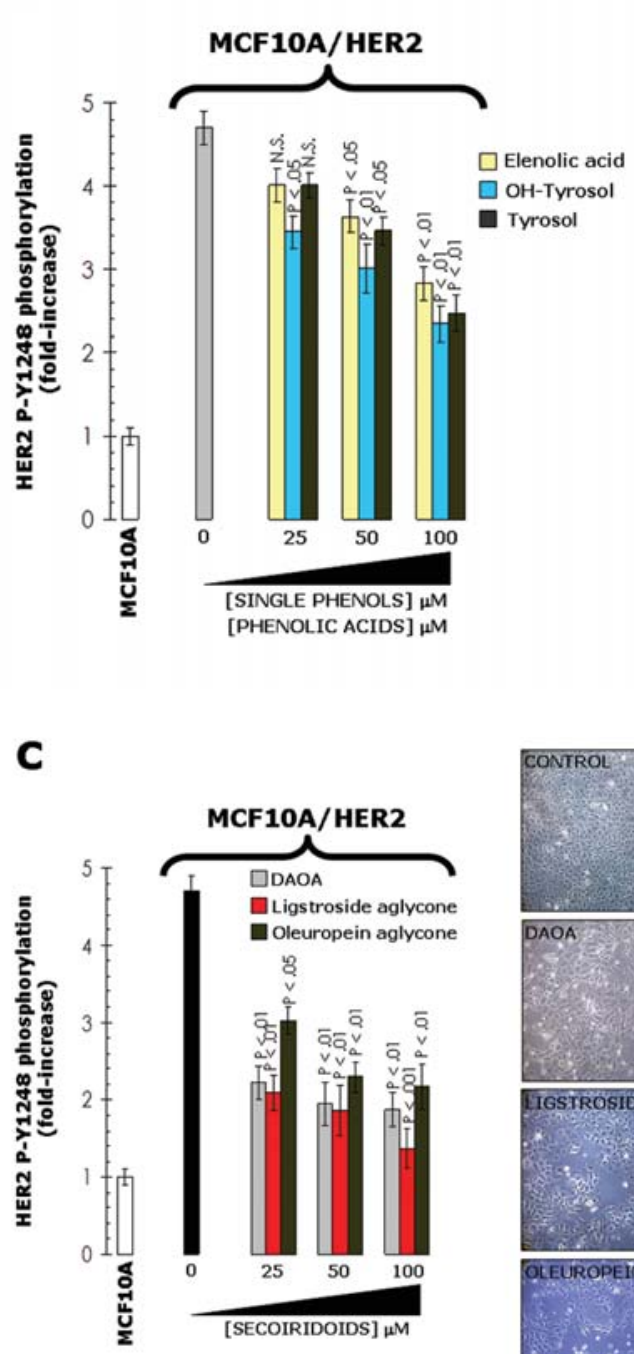
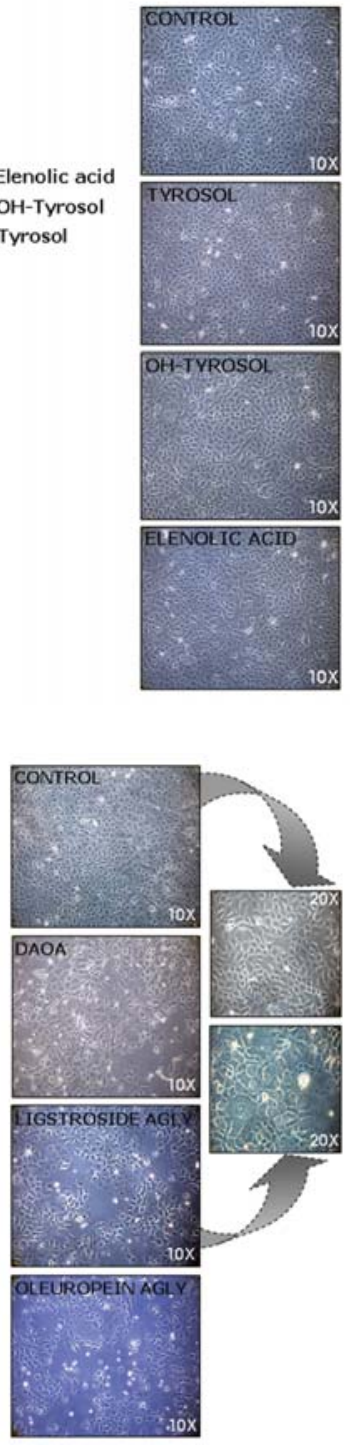

b
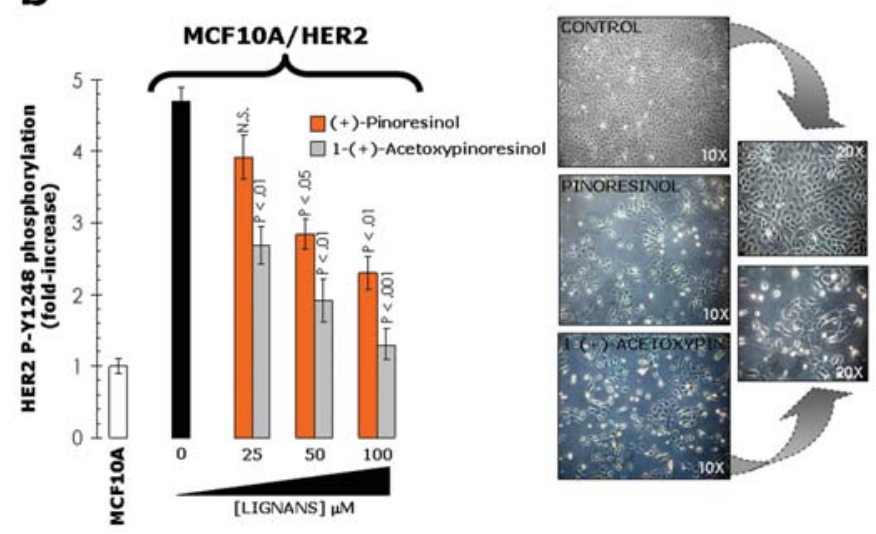

Figure 2. Effects of EVOO single phenols (a), EVOO lignans (b) and EVOO secoiridoids (c) on the activation status of HER2 tyrosine kinase. Overnight serum-starved MCF10A/HER2 cells were cultured in DMEM/F12 medium$0.1 \%$ horse serum in the absence or presence of increasing concentrations of EVOO phenolics for 6 and $24 \mathrm{~h}$. Assessment of the active/inactive status of the HER2 tyrosine kinase receptor was performed by semi-quantitatively determining the degree of degree of phosphorylation of the 1248 tyrosine residue (Tyr 1248) of HER2 by using the FACE ErbB-2 (Y1248) kit as described in 'Materials and methods'. The total HER2 antibody supplied in the FACE ErbB-2 kit allows determining HER2 phosphorylation relative to the total HER2 protein found in the cells. Data were plotted after correction for cell number (performed through use of crystal violet staining) and the measurement of phosphor-HER2 (Y1248) in untreated HER2-negative MCF10A cells was arbitrarily designed as 1.0 -fold. Data are the mean (columns) and 95\% confidence intervals (bars) of three independent experiments performed in duplicate. One-factor ANOVA was used to analyze differences in the relative levels of phosphor-HER2 (Y1248) in MCF10A/ HER2 cells following $6 \mathrm{~h}$ treatment with EVOO phenolics. Statistically significant differences (one-factor ANOVA analysis) between experimental conditions and unsupplemented control cells are labeled. All statistical tests were two-sided. N.S, Not statistically significant. Figure also shows the impact of exogenous supplementation with EVOO phenolics on cell morphology of MCF10A/HER2 cells as assessed by phase contrast microscopic analysis. 


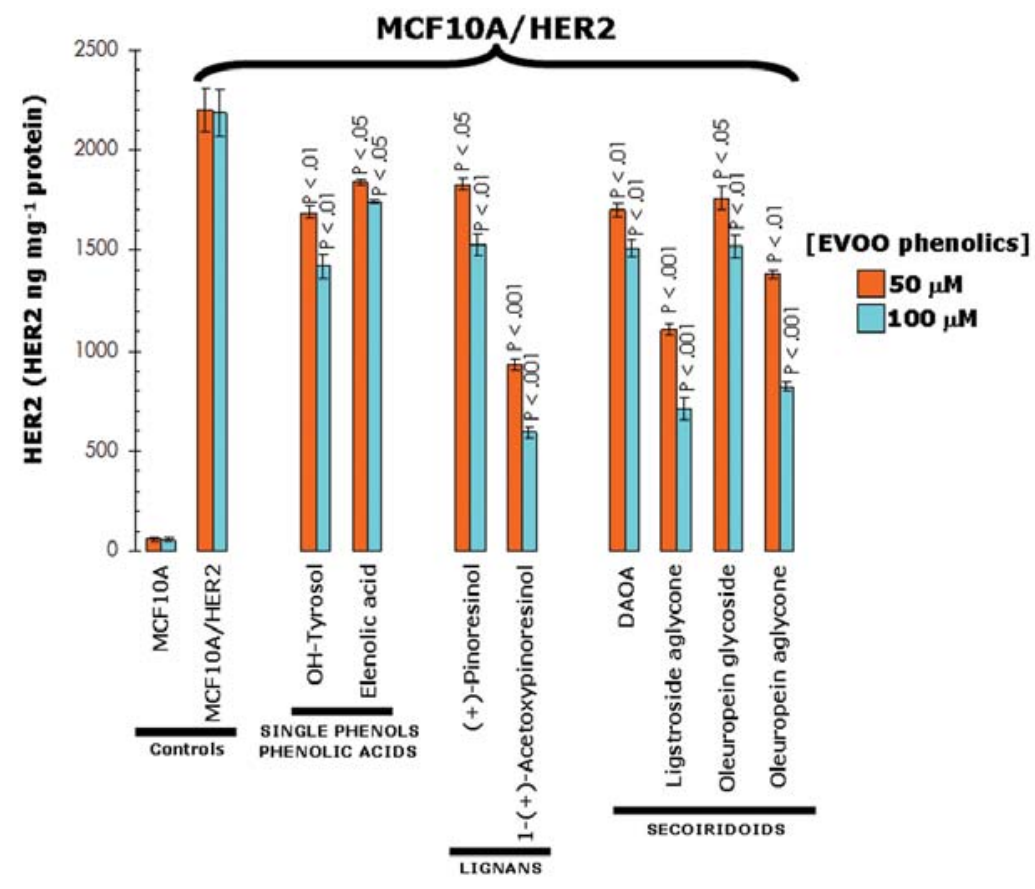

Figure 3. Effects of EVOO phenolics on ectopically driven overexpression of HER2 protein in MCF10A/HER2 cells. Overnight serum-starved MCF10A/ HER2 cells were cultured in DMEM/F12 medium- $0.1 \%$ HS in the absence or presence of increasing concentrations of EVOO phenolics for $48 \mathrm{~h}$. The Oncogene Science HER2 microtiter ELISA was used according to the manufacturer's instructions to compare HER2 protein concentrations in whole cell lysates from EVOO phenolics-treated and untreated control cells. Results are means (columns) and 95\% confidence intervals (bars) of three independent experiments made in triplicate. Statistically significant differences (one-factor ANOVA analysis) between experimental conditions and unsupplemented control cells are labeled. All statistical tests were two-sided.

striking morphological changes (Fig. 2a-c). The cells grew less dense, were significantly bigger in volume and showed a profound reorganization of cell-cell contacts with the appearance of multiple extrusions. Exposure to 1-(+)acetoxypinoresinol rapidly inhibited the growth of MCF10A/ HER2 cells and resulted in cell morphologies compatible with apoptotic cell death (Fig. 2b).

EVOO-derived phenolics down-regulate HER2 protein expression. We then assessed whether long-term exposure (i.e. $48 \mathrm{~h}$ ) to EVOO phenolics not only reduced HER2 tyrosine kinase activity but further affected the cellular content of HER2 protein itself (Fig. 3). Hydroxytyrosol (100 $\mu \mathrm{M})$ reduced HER 2 protein by $35 \%$ in MCF10A/HER 2 cells. Among EVOO-derived lignans, (+)-pinoresinol was significantly less effective at reducing HER2 protein expression than 1-(+)acetoxypinoresinol ( $\sim 30$ vs. $>70 \%$ reduction, respectively). Among EVOO secoiridoids, ligstroside aglycone and oleuropein aglycone were the most active inhibitors of HER2 expression in MCF10A/HER2 cells, with reductions of 68 and $63 \%$, respectively.

Exogenous supplementation with EVOO polyphenols triggers high levels of apoptotic cell death in MCF10A/HER2 cells. The above results, altogether, strongly suggested that EVOOderived might exert tumoricidal effects closely related to the absence/presence of an active HER2-driven oncogenic signaling in breast epithelial cells. Upon treatment with EVOO phenolics, MCF10A cells engineered to stably overexpress HER2 (MCF10A/HER2 cells) likewise exhibited significantly higher degrees of apoptotic cell death than non-cancerous HER2-negative MCF10A/pBABE matched control cells (Fig. 4). This HER2-related sensitivity to the apoptotic effects of EVOO phenolics was mainly observed with EVOO lignans and secoiridoids. Thus, forced expression of HER2 in MCF10A cells increased by $\sim 4$-fold the apoptotic effects of the EVOO lignan 1-(+)-acetoxypinoresinol. HER2 overexpression further promoted an exacerbated sensitivity to the apoptotic effects of the EVOO secoiridoids DAOA, ligstroside aglycone and oleuropein aglycone, with all of them being 2- to 4-times more effective in MCF10A/HER2 cells than in HER2negative MCF10A/pBABE matched control cells (Fig. 4).

EVOO-derived polyphenols inhibit HER2-induced in vitro transformed phenotype. Since growth in soft agar is a canonical oncogenic property, we finally examined the tumoricidal effects of EVOO-derived polyphenols in a soft-agar colony formation assay, which simultaneously measures both anchorage-independent proliferation and cell survival (Fig. 5). Colony formation semi-solid assays in soft-agar likewise revealed the ability of wild-type HER2 to confer anchorageindependency when overexpressed in MCF10A cells (number of colonies $=381 \pm 40$ in MCF10A/HER2 vs. $28 \pm 10$ in MCF10A/pBABE matched control cells; $\mathrm{P}<0.001)$. The EVOO secoiridoid ligstroside aglycone $(50 \mu \mathrm{M})$ significantly reduced both the number (number of colonies $=109 \pm 22$ ) and the size of HER2-induced soft-agar colonies. Remarkably, MCF10A/HER2 cells were incapable to form colonies in soft-agar assays when they were cultured in the presence of the EVOO lignan 1-(+)-acetoxypinoresinol (50 $\mu \mathrm{M}$; number 


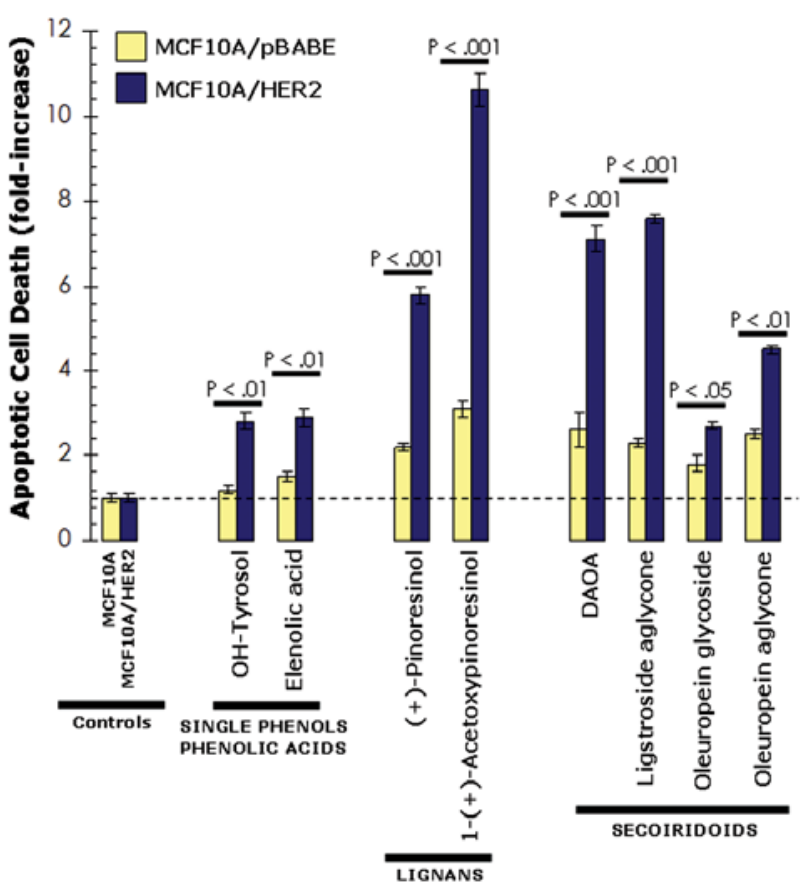

Figure 4. Apoptotic effects of EVOO phenolics in non-cancerous MCF10A breast epithelial cells engineered to overexpress HER2. Quantification of apoptosis-related cell death in MCF10A/HER2 and MCF10A/pBABE matched control cells treated with increasing concentrations of EVOO phenolics was determined by Cell Death ELISA as described in 'Materials and methods'. The enrichment of histone-DNA fragments EVOO phenolicstreated cells was expressed as fold-increase in absorbance by comparing with control (vehicle-treated) cells using the following formula: $\left(\mathrm{A}_{405}\right.$ $\left.\mathrm{A}_{490}\right)_{\text {TREATED }} /\left(\mathrm{A}_{405}-\mathrm{A}_{490}\right)_{\text {UNTREATED }}$. Data are the mean (columns) and $95 \%$ confidence intervals (bars) of three independent experiments performed in duplicate. One-factor ANOVA was used to analyze differences in the percentage of apoptosis between HER2-negative MCF10A cells and HER2positive MCF10A/HER2 cells. Statistically significant differences (onefactor ANOVA analysis) are labeled. All statistical tests were two-sided.

of colonies $=24 \pm 6)$. Indeed, 1-(+)-acetoxypinoresinol-treated MCF10A/HER2 cells behaved as HER2-negative MCF10A/ pBABE control cells.

\section{Discussion}

We designed a systematic approach to investigate the tumoricidal and the anti-HER2 effects of EVOO-derived single phenols (i.e. tyrosol and hydroxytyrosol), phenolic acids (i.e. elenolic acid), lignans [i.e. (+)-pinoresinol and 1-(+)-acetoxypinoresinol] and secoiridoids (i.e. deacetoxy oleuropein aglycone-DAOA-, oleuropein aglycone and ligstroside aglycone) directly extracted from EVOO. First, solid phase extraction followed by semi-preparative HPLC was used to isolate phenolic fractions from commercial EVOO. Second, analytical capillary electrophoresis coupled to mass spectrometry (CE-MS) was performed to check for the composition of the isolated phenolic compounds and to confirm their identity. Third, we evaluated the effects of individual EVOO phenolic on the activation status and expression level of HER2 oncoprotein. Fourth, we assessed the effects of EVOO phenolics on HER2-dependent breast cancer cell proliferation and survival. To determine the biological effects of EVOO-derived phenolics on the functionality of HER2-driven oncogenic signaling in breast cancer disease, we took advantage of the MCF10A cells, a non-transformed, near diploid, spontaneously immortalized human mammary epithelial cell line. These cells provide a useful in vitro system to analyze the effect of the expression of specific genes that have been involved in breast cancer development in a nontransformed genetic background (43-45). In addition to expressing very low levels of HER2, MCF10A cells express high levels of HER1 (EGFR) and are dependent on EGF for optimal growth. Hence, by overexpressing HER 2 in these cells, a common clinical observation of HER1 and HER2 overexpression is recapitulated, and with the addition of EGF, the paracrine/autocrine stimulation of HER receptors-driven oncogenic signalling. Indeed, MCF10A cells acquire both an in vitro transformed and in vivo tumorigenic phenotype following transfection with appropriate vectors containing the HER2 gene (43-45).

Among EVOO phenolics tested, the EVOO-derived polyphenols lignans and secoiridoids were the most potent phytochemicals in decreasing HER2 tyrosine kinase activity (Fig. 6a). Indeed, the $\mathrm{IC}_{50}$ values (i.e. the concentrations of EVOO phenolics needed to decrease HER2 Y1248 phosphorylation by $50 \%$ ) were as low as $64,30,45,24$ and $10 \mu \mathrm{M}$ in the presence of (+)-pinoresinol, 1-(+)-acetoxypinoresinol, oleuropein aglycone, DAOA and ligstroside aglycone, respectively. Conversely, concentrations as high as $100 \mu \mathrm{M}$ were necessary to block HER2 Y1248 phosphorylation by $50 \%$ when using tyrosol, hydroxytyrosol and elenolic acid instead of EVOO polyphenolic molecules. The fact that EVOO-derived lignans and secoiridoids were significantly

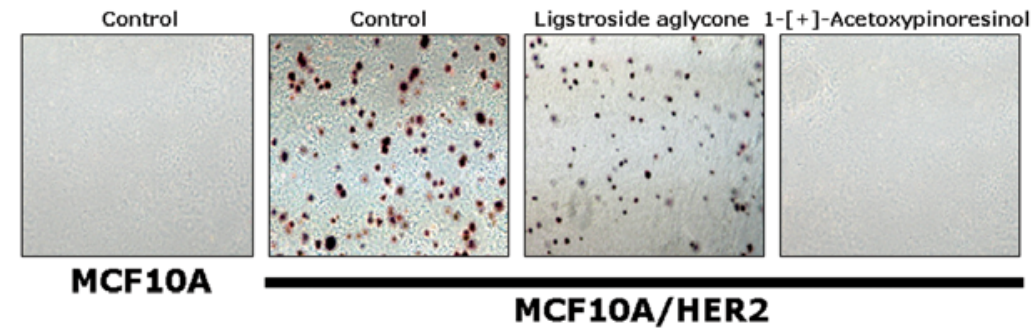

Figure 5. Effects of the EVOO polyphenols on the transforming ability of HER2. MCF10A/HER2 and MCF10A/pBABE matched control cells (10,000 per well) were seeded in 35-mm multi-well plates in culture medium containing $0.35 \%$ low-melting agarose over a $0.7 \%$ agarose basal layer and incubated for 14 days at $37^{\circ} \mathrm{C}$ in a humidified $95 \% \mathrm{O}_{2} 5 \% \mathrm{CO}_{2}$ atmosphere in DMEM/F12 medium supplemented with $10 \%$ horse serum $+20 \mathrm{ng} / \mathrm{ml} \mathrm{EGF}$ in the absence or presence of $50 \mu \mathrm{M}$ ligstroside aglycone and $50 \mu \mathrm{M} 1-(+)$-acetoxypinoresinol. Colonies were then stained with $p$-iodonitrotetrazolium violet (1 mg/ml stock diluted 1:500) for $18 \mathrm{~h}$. Colonies $>50 \mu \mathrm{m}$ in diameter were counted (see representative microphotographs). 


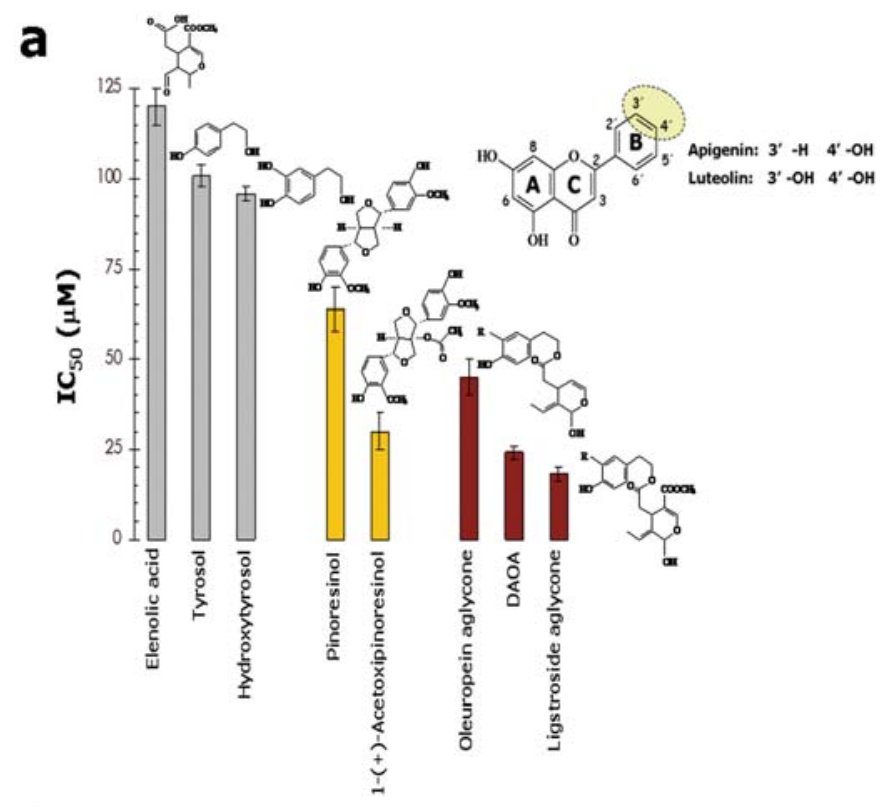

b

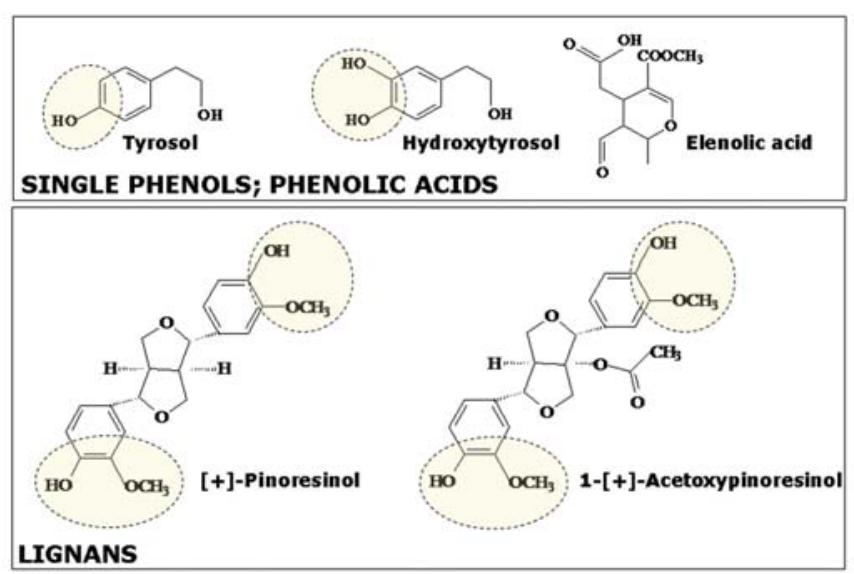

C

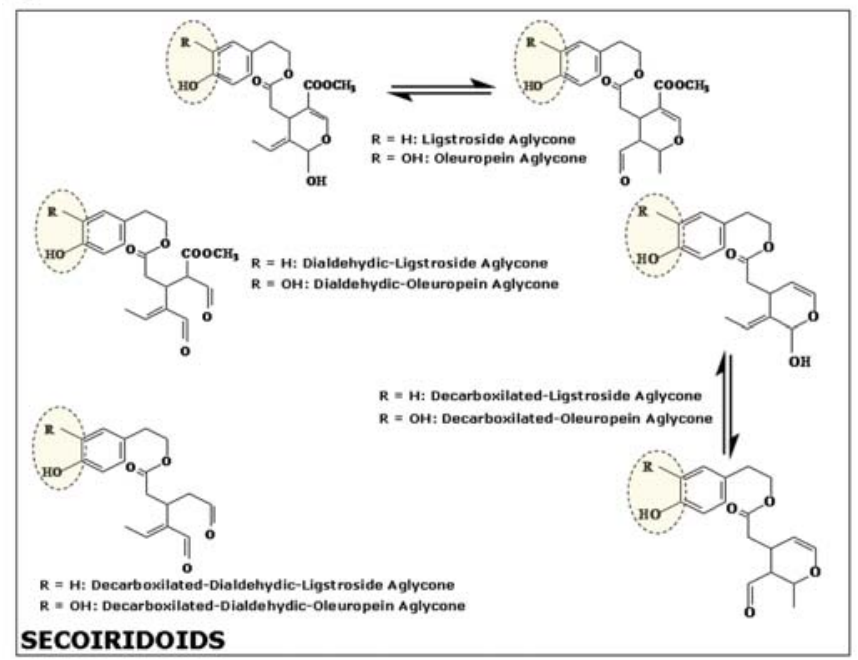

Figure 6. EVOO phenolics inhibitory potencies against HER2 tyrosine kinase activity. Preliminary assessment of structure-activity relationship (SAR). Figure shows the $\mathrm{IC}_{50}$ values (i.e. the concentrations of EVOO phenolics needed to decrease HER2 Y1248 phosphorylation by 50\%) for EVOO phenolics (a). Data are the mean (columns) and 95\% confidence intervals (bars) of three independent experiments performed in duplicate. Fig. also shows the stereochemistry of each EVOO phenolic and the putative structure-activity relationship based on the well-characterized anti-tyrosine kinase activity of the flavonoids apigenin and luteolin (b and c). more effective than EVOO-derived single phenols and phenolic acids to inhibit HER2-associated tyrosine kinase activity strongly suggests that EVOO phenolic compounds with a simple structure (i.e. involving only a single phenol ring) cannot exert inhibitory effects against the tyrosine kinase domain of HER2, and that a more complex structure (i.e. involving two or more phenol rings) is required to efficiently block HER2 tyrosine kinase activity. Indeed, our current findings definitely support the notion that the ability of naturally occurring polyphenols to potently inhibit several kinases involved in signal transduction, mainly protein kinase $\mathrm{C}$ and tyrosine kinases including HER2 largely depend on their chemical structure (31-33). These studies have shown that the position and number of the hydroxyl group on the 2-phenyl ring strongly influence the conformation of the polyphenolic molecules which, in turn, dictate their inhibitory effects. Thus, the introduction of hydroxyl groups on the flavone ring significantly increases the inhibitory potency of flavonoids such as luteolin. The fact that polyhydoxylated flavonols are by far the most effective kinase inhibitors whereas the replacement of hydroxyl groups with methoxyl groups yields much weaker kinase inhibitors clearly reveal the importance of hydrogen bond between flavonoids and the target kinase. When considering the stereochemistry of flavonoids such as apigenin and luteolin, which actually work as effective phosphotyrosine-receptor kinase blockers by preventing ATP from binding to the tyrosine kinase domain of HER2 (31-33), it is reasonable to suggest that preliminary structure-activity relationship (SAR) should focus on the hydroxylated nature of the external phenolic rings of EVOO-derived lignans and secoiridoids (Fig. 6b and c).

We found that EVOO polyphenols not only repressed the phosphor tyrosine levels of HER2 (short-term exposure) but further depleted HER2 protein itself (long-term exposure). Although the definite molecular mechanism underlying EVOO polyphenols-induced blockade of HER2 tyrosine kinase activity and down-regulation of HER2 protein expression was beyond the scope of this study, it is reasonable to suggest that the anti-HER2 actions of the EVOO polyphenols lignans and secoiridoids do no relate to the cellular transcriptional machinery that controls the endogenous HER2 locus in breast cancer cells because they drastically decreased the content of HER2 protein in MCF10A cells engineered to ectopically overexpress HER2 under control of a viral promoter. Moreover, HER2-overexpressing breast epithelial cells exhibited an exacerbated sensitivity to EVOO polyphenols-induced cell damage further supporting the notion that their tumoricidal mode of action largely depend on their ability to impair HER2 activity and expression. Similarly to the ability of other transforming proteins studied in MCF10A cells, the forced expression of HER2 may induce an epithelial to mesenchymal transition associated with a more malignant phenotype. It is well established that MCF10A cells are not anchorage-independent in the absence of oncogenic stimuli and colony formation of MCF10A cells observed in soft agar, if any, represents the background level for the colony formation experiment. Consistent with a role for HER2 in the regulation of anchorage independence MCF10A cells engineered to overexpress HER2 likewise 
showed significant growth in soft agar, while the vector control did not. Thus, overexpression of constitutively active HER2 is sufficient for malignant transformation. Importantly, MCF10A/HER2 cells failed to fully exhibit a transformed phenotype when cultured in the presence of EVOO-derived lignans and secoiridoids. Thus, exogenous supplementation with the lignan 1-(+)-acetoxy-pinoresinol and the secoiridoid ligstroside aglycone was sufficient to strongly inhibit HER2-induced anchorage-independent growth and to potently prevent the transformed morphology of HER2-overexpressing MCF10A cells growing upon semisolid conditions.

In summary, this study has identified novel roles for naturally-occurring EVOO-derived polyphenols in human breast cancer cell growth and HER2-regulated malignant transformation. These findings have provided new insights into the molecular mechanisms underlying the protective effects of naturally occurring EVOO biocompounds on breast cancer risk. This elucidation of the strong inhibitory effects of EVOO-derived lignans and secoiridoids against the oncogenic activity of the tyrosine kinase receptor HER2, together with the fact that humans have safely been ingesting significant amounts of lignans and secoiridoids as long as they have been consuming olives and EVOO, strongly suggest that the stereochemistry of these polyphenols might provide an excellent and safe platform for the design of novel HER2-targeted anti-cancer drugs.

\section{Acknowledgements}

Javier A. Menendez is the recipient of a Basic, Clinical and Translational Research Award (BCTR0600894) from the Susan G. Komen Breast Cancer Foundation (TX, USA). This study was supported in part by Instituto de Salud Carlos III (Ministerio de Sanidad y Consumo, Fondo de Investigación Sanitaria -FIS-, Spain, Grants CP05-00090, PI06-0778 and RD06-0020-0028 to Javier A. Menendez). Javier A. Menendez and Antonio Segura-Carretero are also supported by a Grant from the Fundación Científica de la Asociación Española Contra el Cáncer (AECC, Spain) and by the Ministerio de Educación y Ciencia CTQ2005-01914/ BQU and Junta de Andalucía (Proyecto de Excelencia AGR-02619).

\section{References}

1. Trichopoulou A, Lagiou P, Kuper H and Trichopoulos D: Cancer and Mediterranean dietary traditions. Cancer Epidemiol Biomarkers Prev 9: 869-873, 2000.

2. Simopoulos AP: The traditional diet of Greece and cancer. Eur J Cancer Prev 13: 219-230, 2004

3. Perez-Jimenez F, Alvarez de Cienfuegos G, Badimon L, Barja G, Battino M, Blanco A, Bonanome A, Colomer R, Corella-Piquer D, Covas I, Chamorro-Quiros J, Escrich E, Gaforio JJ, Garcia Luna PP, Hidalgo L, Kafatos A, Kris-Etherton PM, Lairon D, Lamuela-Raventos R, Lopez-Miranda J, Lopez-Segura F, Martinez-Gonzalez MA, Mata P, Mataix J, Ordovas J, Osada J, Pacheco-Reyes R, Perucho M, Pineda-Priego M, Quiles JL, Ramirez-Tortosa MC, Ruiz-Gutierrez V, Sanchez-Rovira P, Solfrizzi V, Soriguer-Escofet F, de la Torre-Fornell R, Trichopoulos A, Villalba-Montoro JM, Villar-Ortiz JR and Visioli F: International conference on the healthy effect of virgin olive oil. Eur J Clin Invest 35: 421-424, 2005.

4. Colomer R and Menendez JA: Mediterranean diet, olive oil and cancer. Clin Transl Oncol 8: 15-21, 2006.
5. Colomer R, Lupu R, Papadimitropoulou A, Vellón L, Vázquez-Martín A, Brunet J, Fernández-Gutiérrez A, Segura-Carretero A and Menéndez JA: Giacomo Castelvetro's salads. Anti-HER2 oncogene nutraceuticals since the 17th century? Clin Transl Oncol 10: 30-34, 2008.

6. Menendez JA and Lupu R: Mediterranean dietary traditions for the molecular treatment of human cancer: anti-oncogenic actions of the main olive oil's monounsaturated fatty acid oleic acid (18:1n-9). Curr Pharm Biotechnol 7: 495-502, 2006.

7. Solanas M, Hurtado A, Costa I, Moral R, Menendez JA, Colomer R and Escrich E: Effects of a high olive oil diet on the clinical behavior and histopathological features of rat DMBAinduced mammary tumors compared with a high corn oil diet. Int J Oncol 21: 745-753, 2002.

8. Moral R, Solanas M, Garcia G, Colomer R and Escrich E: Modulation of EGFR and neu expression by $n-6$ and $n-9$ highfat diets in experimental mammary adenocarcinomas. Oncol Rep 10: 1417-1424, 2003

9. Menendez JA, Ropero S, Lupu R and Colomer R: Dietary fatty acids regulate the activation status of Her-2/neu (c-erbB-2) oncogene in breast cancer cells. Ann Oncol 15: 1719-1721, 2004 .

10. Menendez JA, Vellon L, Colomer R and Lupu R: Oleic acid, the main monounsaturated fatty acid of olive oil, suppresses Her-2/ neu (erbB-2) expression and synergistically enhances the growth inhibitory effects of trastuzumab (Herceptin) in breast cancer cells with Her-2/neu oncogene amplification. Ann Oncol 16: 359-371, 2005.

11. Nelson R: Oleic acid suppresses overexpression of ERBB2 oncogene. Lancet Oncol 6: 69, 2005.

12. Menendez JA, Papadimitropoulou A, Vellon L and Lupu R: A genomic explanation connecting 'Mediterranean diet', olive oil and cancer: Oleic acid, the main monounsaturated fatty acid of olive oil, induces formation of inhibitory 'PEA3 transcription factor-PEA3 DNA binding site' complexes at the Her-2/neu (erbB-2) oncogene promoter in breast, ovarian and stomach cancer cells. Eur J Cancer 42: 2425-2432, 2006.

13. Menendez JA, Vazquez-Martin A, Colomer R, Brunet J, Carrasco-Pancorbo A, Garcia-Villalba R, Fernandez-Gutierrez A and Segura-Carretero A: Olive oil's bitter principle reverses acquired autoresistance to trastuzumab (Herceptin) in HER2overexpressing breast cancer cells. BMC Cancer 7: 80, 2007.

14. Sant M, Allemani C, Sieri S, Krogh V, Menard S, Tagliabue E, Nardini E, Micheli A, Crosignani P, Muti P and Berrino F: Salad vegetables dietary pattern protects against HER-2-positive breast cancer: A prospective Italian study. Int J Cancer 121: 911-914, 2007.

15. Harari D and Yarden Y: Molecular mechanisms underlying ErbB2/HER2 action in breast cancer. Oncogene 19: 6102-6114, 2000.

16. Yarden Y: Biology of HER2 and its importance in breast cancer. Oncology 61 (Suppl 2): 1-13, 2001.

17. Yarden Y and Sliwkowski MX: Untangling the ErbB signalling network. Nat Rev Mol Cell Biol 2: 127-137, 2001.

18. Hynes NE and Lane HA: ERBB receptors and cancer: the complexity of targeted inhibitors. Nat Rev Cancer 5: 341-354, 2005.

19. Nahta R, Yu D, Hung MC, Hortobagyi GN and Esteva FJ: Mechanisms of disease: understanding resistance to HER2targeted therapy in human breast cancer. Nat Clin Pract Oncol 3: 269-280, 2006

20. Escrich E, Solanas M, Moral R, Costa I and Grau L: Are the olive oil and other dietary lipids related to cancer? Experimental evidence. Clin Transl Oncol 8: 868-883, 2006.

21. Escrich E, Moral R, Grau L, Costa I and Solanas M: Molecular mechanisms of the effects of olive oil and other dietary lipids on cancer. Mol Nutr Food Res 51: 1279-1292, 2007.

22. Owen RW, Giacosa A, Hull WE, Haubner R, Spiegelhalder B and Bartsch H: The antioxidant/anticancer potential of phenolic compounds isolated from olive oil. Eur J Cancer 36: 1235-1247, 2000 .

23. Visioli F and Galli C: Olive oil: more than just oleic acid. Am J Clin Nutr 72: 853, 2000.

24. Beauchamp GK, Keast RS, Morel D, Lin J, Pika J, Han Q, Lee CH, Smith $\mathrm{AB}$ and Breslin PA: Phytochemistry: ibuprofen-like activity in extra-virgin olive oil. Nature 437: 45-46, 2005.

25. Fogliano V and Sacchi R: Oleocanthal in olive oil: between myth and reality. Mol Nutr Food Res 50: 5-6, 2006. 
26. Galli $\mathrm{C}$ and Visioli F: Antioxidant and other activities of phenolics in olives/olive oil, typical components of the Mediterranean diet. Lipids 34 (Suppl): 23-26, 1999.

27. Visioli F, Poli A and Gall C: Antioxidant and other biological activities of phenols from olives and olive oil. Med Res Rev 22: $65-75,2002$

28. Visioli F and Galli C: Phenolics from olive oil and its waste products. Biological activities in in vitro and in vivo studies. World Rev Nutr Diet 88: 233-237, 2001.

29. Visioli F and Galli C: Biological properties of olive oil phytochemicals. Crit Rev Food Sci Nutr 42: 209-221, 2002.

30. Owen RW, Mier W, Giacosa A, Hull WE, Spiegelhalder B and Bartsch H: Identification of lignans as major components in the phenolic fraction of olive oil. Clin Chem 46: 976-988, 2000.

31. Way TD, Kao MC and Lin JK: Apigenin induces apoptosis through proteasomal degradation of HER2/neu in HER2/neuoverexpressing breast cancer cells via the phosphatidylinositol 3-kinase/Akt-dependent pathway. J Biol Chem 279: 4479-4489, 2004.

32. Way TD, Kao MC and Lin JK: Degradation of HER2/neu by apigenin induces apoptosis through cytochrome c release and caspase-3 activation in HER2/neu-overexpressing breast cancer cells. FEBS Lett 579: 145-152, 2005.

33. Chiang CT, Way TD and Lin JK: Sensitizing HER2overexpressing cancer cells to luteolin-induced apoptosis through suppressing p21WAF1/CIP1 expression with rapamycin. Mol Cancer Ther 6: 2127-2138, 2007.

34. Shimizu M, Deguchi A, Joe AK, Mckoy JF, Moriwaki H and Weinstein IB: EGCG inhibits activation of HER3 and expression of cyclooxygenase-2 in human colon cancer cells. J Exp Ther Oncol 5: 69-78, 2005.

35. Shimizu M, Deguchi A, Hara Y, Moriwaki H and Weinstein IB: EGCG inhibits activation of the insulin-like growth factor-1 receptor in human colon cancer cells. Biochem Biophys Res Commun 334: 947-953, 2005.

36. Shimizu M, Deguchi A, Lim JT, Moriwaki H, Kopelovich L and Weinstein IB: (-)-Epigallocatechin gallate and polyphenon $\mathrm{E}$ inhibit growth and activation of the epidermal growth factor receptor and human epidermal growth factor receptor-2 signaling pathways in human colon cancer cells. Clin Cancer Res 11: 2735-2746, 2005.

37. Gomez Caravaca AM, Carrasco Pancorbo A, Canabate Diaz B, Segura Carretero A and Fernandez Gutierrez A: Electrophoretic identification and quantitation of compounds in the polyphenolic fraction of extra-virgin olive oil. Electrophoresis 26: 3538-3551, 2005.
38. Carrasco-Pancorbo A, Cerretani L, Bendini A, Segura-Carretero A, Del Carlo M, Gallina-Toschi T, Lercker G, Compagnone D and Fernandez-Gutierrez A: Evaluation of the antioxidant capacity of individual phenolic compounds in virgin olive oil. J Agric Food Chem 53: 8918-8925, 2005.

39. Carrasco-Pancorbo A, Arraez-Roman D, Segura-Carretero A and Fernandez-Gutierrez A: Capillary electrophoresiselectrospray ionization-mass spectrometry method to determine the phenolic fraction of extra-virgin olive oil. Electrophoresis 27: 2182-2196, 2006

40. Carrasco-Pancorbo A, Gomez-Caravaca AM, Cerretani L, Bendini A, Segura-Carretero A and Fernandez-Gutierrez A: Rapid quantification of the phenolic fraction of Spanish virgin olive oils by capillary electrophoresis with UV detection. J Agric Food Chem 54: 7984-7991, 2006.

41. Carrasco-Pancorbo A, Neusüss C, Pelzing M, Segura-Carretero A and Fernández-Gutiérrez A: CE- and HPLC-TOF-MS for the characterization of phenolic compounds in olive oil. Electrophoresis 28: 806-821, 2007.

42. Bendini A, Cerretani L, Carrasco-Pancorbo A, Gómez-Caravaca AM, Segura-Carretero A, Fernández-Gutiérrez A and Lercker G: Phenolic molecules in virgin olive oils: a survey of their sensory properties, health effects, antioxidant activity and analytical methods. An overview of the last decade. Molecules 12: 1679-1719, 2007.

43. Ciardiello F, Gottardis M, Basolo F, Pepe S, Normanno N, Dickson RB, Bianco AR and Salomon DS: Additive effects of c-erbB-2, c-Ha-ras, and transforming growth factor-alpha genes on in vitro transformation of human mammary epithelial cells. Mol Carcinog 6: 43-52, 1992.

44. Giunciuglio D, Culty M, Fassina G, Masiello L, Melchiori A, Paglialunga G, Arand G, Ciardiello F, Basolo F and Thompson EW: Invasive phenotype of MCF10A cells overexpressing c-Ha-ras and c-erbB-2 oncogenes. Int J Cancer 63: 815-822, 1995.

45. Ciardiello F, Caputo R, Pomatico G, De Laurentiis M, De Placido S, Bianco AR and Tortora G: Resistance to taxanes is induced by c-erbB-2 overexpression in human MCF-10A mammary epithelial cells and is blocked by combined treatment with an antisense oligonucleotide targeting type I protein kinase A. Int J Cancer 85: 710-715, 2000. 